\title{
Sustainable Strategic Urban Planning: Methodology for Urban Renovation at district level
}

\author{
Estefanía Vallejo ${ }^{1}$, Cristina Criado ${ }^{2}$, Eneko Arrizabalaga ${ }^{3}$ and Ali Vasallo ${ }^{1}$ \\ 1 CARTIF Foundation, Boecillo (Spain) \\ 2 ACCIONA Construction, Madrid (Spain) \\ 3 TECNALIA Research and Innovation, Bilbao (Spain)
}

\begin{abstract}
Sustainable urban renovation is characterized by multiple factors (e.g. technical, socio-economic, environmental and ethical perspectives), different spatial scales and a number of administrative structures that should address the evaluation of alternative scenarios or solutions. This defines a complex decision problem that includes different stakeholders where several aspects need to be considered simultaneously. In spite of the knowledge and experiences during the recent years, there is a need of methods that lead the decision-making processes.

In response, a methodology based on the global idea and implications of working towards a more sustainable and energy efficient cities as a holistic procedure for urban renovation at district level is proposed in the European Smart City project CITyFiED. The methodology has the energy efficiency as main pillar and the local authorities as client. It is composed of seven phases that ensures an effective dialogue among all the stakeholders, aiming to understand the objectives and needs of the city to define a set of Strategies for Sustainable Urban Renovation and their integration within the Strategic Urban Planning of the cities.
\end{abstract}

Keywords: Sustainability, Decision making, Urban Renovation, Indicators, Energy planning

\section{Introduction}

Urban planning and regeneration process are concepts that involve a number of dimensions, e.g. technical, socioeconomic, environmental and ethical perspectives. Sustainable urban regeneration is characterized by multiple factors, different spatial scales and a number of administrative structures that should address the evaluation of alternative scenarios or solutions. This defines a complex decision problem that includes a number of different stakeholders where several aspects need to be considered simultaneously. It is usually based on series of qualitative and quantitative data related to empirical observations, physical conditions and trends. In the decisionmaking process, cities need to invest a great deal of resources and time in the collection of information to deliver such assessments and decisions. However, very little is known about how decisions are made and the processes that lead to them.

There is a need to better identify, analyse, model and map resources and the current situation of urban settlements and solutions to achieve a more efficient built environment and match its demands with efficient sustainable energy sources at the least cost. Such energy planning is already mandated in the Energy Efficiency Directive (Directive 2012/27/EU). This needs to be done at local, regional and national levels to help develop energy strategies and ensure their consistency at national level and with EU policies; in fact, promoting sustainable urban development is a key element of the European Cohesion Policy and a continuous process.

During the last years, a great deal of research have been develop on one hand, by Technical Committees and Working Groups, which have been working in the field of Smart Cities, such us ISO TC/268, developing requirements, frameworks, guidance and supporting techniques and tools related to the achievement of sustainable development. On the other hand, there are many EU projects working to create models on energy efficient and sustainable city planning, such as PLEEC, or financial entities, such us The Word Bank, who has developed the Urban Regeneration Decision Tool.

Despite the vast amount of knowledge and tangible experiences that have being generated, there is still a need of deployment of holistic methodologies which comprise a global approach at city level and embrace the concept of sustainability. This implies the development of procedures, tools, key performance indicators and guidelines to 
cover the large scale and complexity of this approach. In this context, under the umbrella of Sustainable Strategic Urban Planning, a novel methodology for urban renovation at district level is proposed and validated by the European Smart City project CITyFiED (Grant Agreement Nº 609129).

\section{CITyFiED Methodology for city renovation at district level}

According to the United Nations, in 2014 more than half of the world's population was living in urban areas and two third of the world's population will be living in an urban area by 2050 (United Nations, 2014) being Europe the most urbanized continent (URBACT, 2015). The forecast for 2050 in this case is to increase the percentage up to 75 percent (Eurostat, 2016). Besides, urban areas are engines of regional and national growth as they generate 53 percent of gross national product (GNP) in low-income countries, 73 percent in middle-income countries, and 85 percent in high-income countries (World Bank, 1999).

These features bring about many desirable advantages for citizens. Although the concentration in cities usually supposes an increase of density and less consumption of resources, cities use two-thirds of the world's energy and generate three-fourths of the world's $\mathrm{CO}_{2}$ emissions (Smart Cities Council, 2013). In addition, urban areas have important drawbacks, being waste production, carbon emissions, pollution, lack of preservation of heritage and environment, traffic congestion, etc. (Broere, 2012). It is analysed that the exposure of citizens to these problems is more than two times higher than in the case of people living in rural areas (Eurostat, 2016).

It seems that residential sector of small and medium sizes cities are the strategic lines of action to solve these problems due to their potential. The total number of dwellings in Europe in 2014 was 249,652.26 thousands and from those, around 45\% were built before 1969, 32\% between 1970 and 1989 and only $9.3 \%$ in the 90's. Moreover, the energy consumption of the residential sector was 263.22 Mtoe what supposes $158.76 \mathrm{kWh} / \mathrm{m}^{2}$, still far from the H2020 set objectives (BPIE database). This data shows an elevated average age of the building stock in Europe with low energy performance and therefore, susceptible of being renovated. On the other hand, an $83 \%$ of cities in Europe are small and medium cities in terms of population (50.000-250.000 inhabitants) (Dijkstra and Poelman, 2012).

Above all, urban lifestyle needs to be sustainable, and sustainability should be understood from three points of view: environmental, socio-economic and institutional. Citizens' current way of life must be reconsidered and cities need to find a way to regenerate themselves in order to ensure long-term sustainability and overcome the current challenges they are facing.

In CITyFiED Methodology, the sustainable city renovation is understood as regeneration of actions, policies, and processes within a city, which address interrelated technical, spatial, and socio-economic problems in order to reduce environmental impact, mitigate environmental risk, and improve environmental quality of urban systems, lifestyles and assets. It aims to enable the replicability and mass-market deployment of energy-efficient retrofit of districts, considering as a reference the large CITyFiED demonstration cases in the cities of Lund (Sweden), Laguna de Duero (Spain) and Soma (Turkey), decision-making processes and business models.

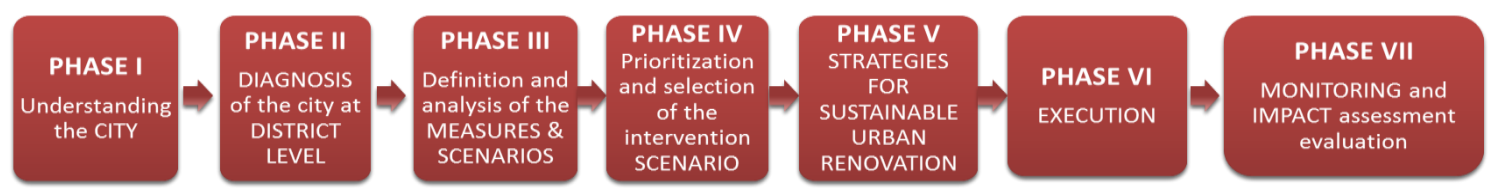

Fig. 1: Phases of CITyFiED Methodology and the twofold scale

The Methodology is a procedure composed of 7 phases and a decision making process that aims to understand the city (objectives and needs) in order to deliver a set of customized Strategies for the Sustainable Urban Renovation at district level with the energy efficiency as the main pillar and local authorities as clients.

Each phase ensures an effective dialogue among all stakeholders and considers ways to strengthen confidence in decision-making processes. The present approach has been validated through the active participation of the CITyFiED network of cities.

The methodological approach foresees the creation of an External Consultancy Group (ECG) to closely cooperate with the local authorities when understanding the city and setting up the most suitable set of strategies for the 
urban renovation at district level. The process is supported by different tools and levels of indicators that would enable the assessment of the suggested actions (Strategies for Sustainable Urban Renovation) as compared to the original situation and objectives.

Three levels of indicators have been defined: City Level Indicators (Level 1) at city \& district level, Project Level Indicators KPIs (Level 2), and Impact Assessment Indicators at city level (Level 3).

\subsection{Involvement of Stakeholders}

The Methodology addresses the roles and responsibilities of the stakeholders involved along the application phases. In the first place, the Municipalities, representing the interest of citizens. They also promote the renovations at city or district levels to increase the sustainable performance of cities. Therefore, the experts representing the Municipality are conceived as collaborative clients in the methodology approach, divided into several committees as they are usually organized.

Public Participation understood as citizens, NGOs, neighbors associations, etc. are other group of stakeholders involved in sustainable renovations through different participation techniques cause "all members of society have a key role in addressing the energy climate challenge with their local authorities" (CoM, 2010).

Besides, External Stakeholders are required to carry out the renovation as constructors, financial entities, energy companies, investors, etc. As one key innovative aspect of the methodology, it proposes the foresight of an External Consultancy Group (ECG) to offer their services and closely cooperate with the Municipality. This is a multidisciplinary consultancy that supports the local authorities when understanding the city and setting up the most suitable set of Strategies for Sustainable Urban Renovation (SSUR), facilitating consequently the decisionmaking process.

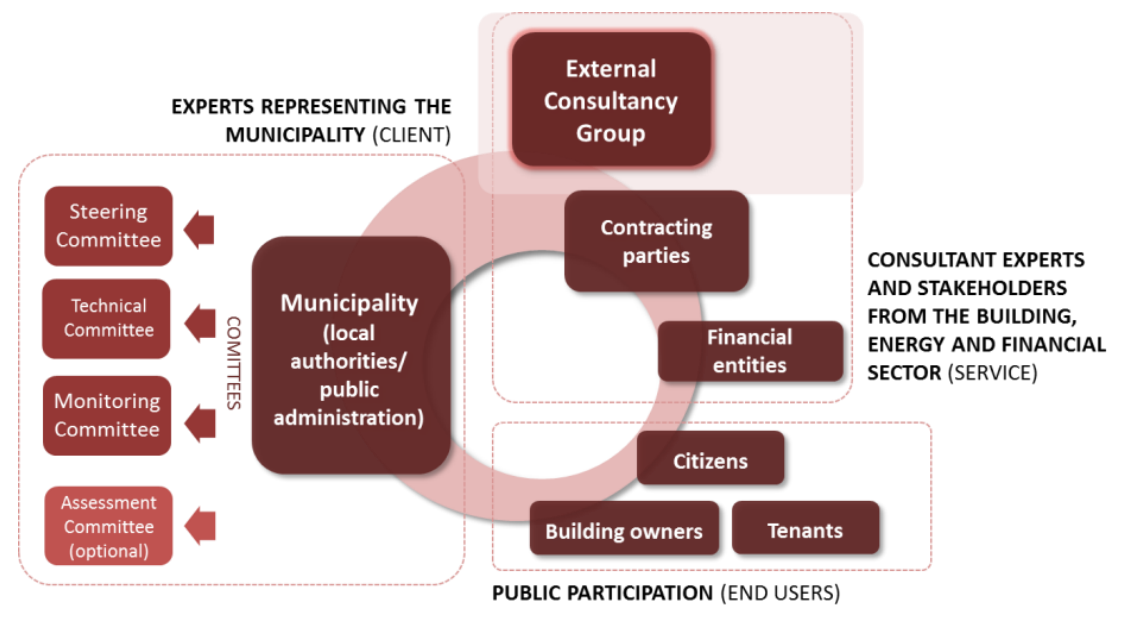

Fig. 2: Stakeholders defined in the Methodology

These stakeholders are sorted in different groups as shown in Fig. 2:

\section{Experts representing the Municipality (Client)}

- Steering committee. This committee includes those capable actors to make decisions in the local authorities' bodies, providing “(...) strategic direction and political support during the process” (CoM, 2010). Their mission is focused on the first stages of the process, i.e., understanding the city and diagnosis, selection of scenarios, etc.

- Technical committee. It is recommended to create this commission from a multi-criteria perspective, including urban planners, technicians, engineers, architects, designers, environmental consultants, etc.

- Monitoring committee. This committee is in charge of the monitoring strategy definition and implementation during the execution and for the final evaluation.

- Advisory committee. Other politicians or technicians from higher levels of the Public Administration could form an additional group of stakeholders to advice the Steering committee, i.e., regional and state technicians. 


\section{Consultant experts and stakeholders from the building, energy and financial sector (Service)}

- External Consultancy Group (ECG). This group is conceived as a multi-perspective consultancy. It consists of technical consultants, technological institutes, research institutions, academia, energy experts, engineers, etc. that assess the Municipality during first phases of the methodology to define the initial situation, evaluate measures and scenarios and define the Strategies for the Sustainable Urban Renovation (SSUR). The addition of this group reduces risks by guaranteeing the achievement of the final objectives

- Contracting parties. These are independent companies and entities involved in the execution of the works that normally should be selected through tender/bidding process (e.g. architecture firms, building and energy companies, etc.). They should establish a fluent dialogue with the technical committee.

- $\quad$ Financial institutions.

\section{Public participation (End users)}

This group is formed by citizens, neighbourhood associations, housing associations, NGOs, etc. Their feedback is collected during all the process, but especially during diagnosis, decision-making and final evaluation. Building owners and tenants are deeply involved in the strategies definition.

\section{Phases of the Methodology}

The Methodology is deployed in seven phases as can be seen in Fig. 3. Each phase ensures an effective dialogue among all the stakeholders previously defined in order to ease the decision-making processes. It combines both district and city scales, starting with the city and district analysis, proposing initiatives at district level and pursuing the impact of the renovation and the accomplishment with the initial objectives at both scales.

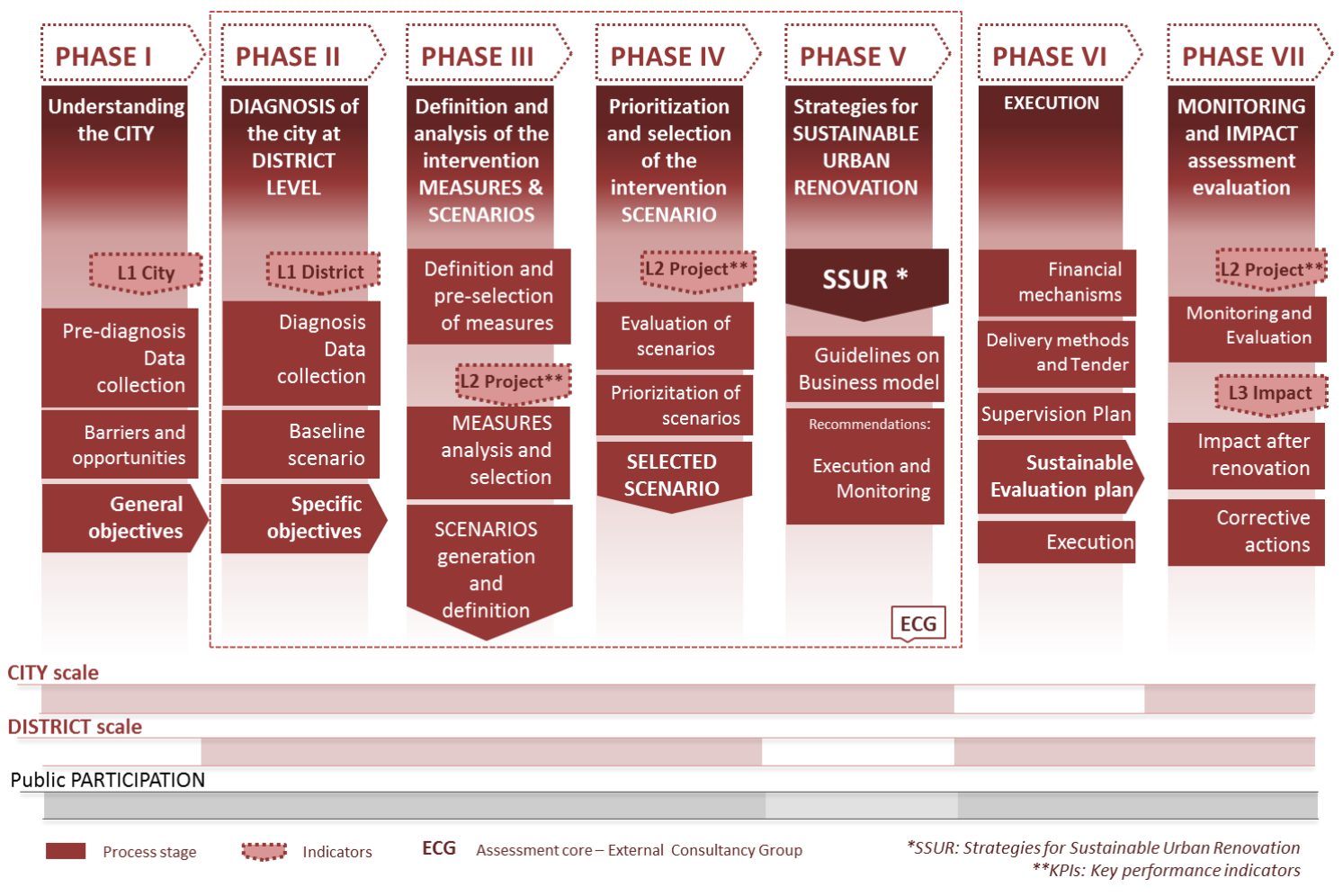

Fig. 3: Methodological approach for urban renovation and planning

\section{Phase I: Understanding the city}

This phase aims to make a first approach to the city understanding, evaluating its context and needs with a set of City Level Indicators as a supporting tool and taking into account public participation. This analysis, combined with the definition of the long-term city vision, enables the identification of the city sustainable pathway within 
its Strategic Urban Planning and the definition of the general objectives that guide this transition.

The pre-diagnosis and data collection of the city is precisely the first step of this phase. Here, the most relevant factors at city level from different perspectives are evaluated in order to contextualize the current situation of the city. The complexity of urban areas, together with the ambitious scope of the methodology and the guidelines for sustainable development from the EC, makes necessary to consider a multi-criteria or holistic perspective during this analysis. However, in order to facilitate the application of the methodology it was necessary to limit the scope of the evaluation. Therefore, considering that the purpose of the CITyFiED project, the matrix of Fig. 4 was defined to gather all the relevant areas to be analyzed. The columns of this matrix are defined as "city strategic areas" which represent the main ambits that will be treated by the implementation of specific improvement interventions at district and city scale. On the other hand, the rows of the matrix are defined as "application areas" and represent the different sectors of the city in which the different interventions can be implemented.

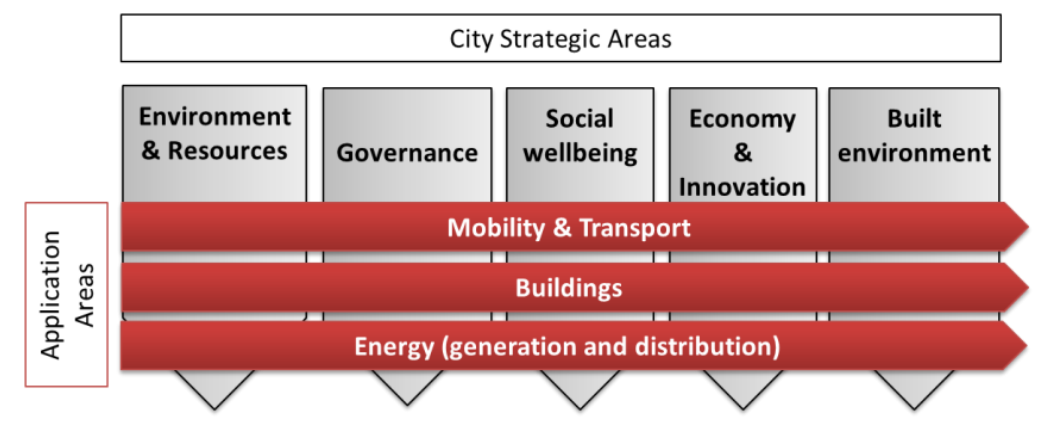

Fig. 4: CITyFiED Application Areas and City Strategic Areas

Following this perspective, the pre-diagnosis will cover aspects of the city such as culture, economy, urban morphology or the main constraints and opportunities for the implementation of measures (legal framework, financial opportunities, etc.). This analysis will contribute to the required initial city context knowledge for the definition of the general objectives of the Strategic Urban Planning.

However, the quantification of specific City Level Indicators (Level 1) that can be compared to other city values provides a better understanding of the improvement potential and needs of different areas of the city evaluated. This is covered by the second step, in which a set of indexes related to the city level analysis "Level 1 City indicators" serve to evaluate the sustainability standards, identify city's strengths and weaknesses, and help setting general and specific objectives for the city.

Although many cities are implementing various low-carbon practices, it is still unclear how this sustainability or low carbon level can be certified. In this context, the methodology developed defines its own set of indicators considering the many efforts that have been previously made at developing indicators-based frameworks capable of evaluating cities' sustainability. The final selection of indicators that at this point includes 17 mobility related indicators, 14 building related indicators and 14 energy related indicators, was carried out following a rigorous internal and external validation process. The internal validation involved CITyFiED project partners (technicians, experts, etc.) and participants involved in the demo sites. The external validation on the other hand, involved the cities of the City Cluster and the Community of Interest, and took part during the Workshop about the CITyFiED Methodology performed in Laguna de Duero (Spain) in March 2017, with approximately 60 attendees.

Besides, each of the defined Level 1 City indicator has its specific reference value defined, considering the average values for the European cities. This allows understanding the distance between the initial situation of the city and the reference value in a way that cities can identify easily the area in which are performing worse than it is supposed to. The complementation of this indicator based analysis with different techniques such as the SWOT analysis and Workshops that include the main stakeholders involved in the planning process will facilitate both the definition of the long-term city vision and their general objectives.

\section{Phase II: Diagnosis of the city at district level}

In this second phase of the methodology the city is understood as an aggregation of districts. Taking into account the results in Phase I and following a bottom-up approach, the city is analyzed at district level to define the specific objectives for the districts object of the intervention. These specific objectives will serve to outline the different 
set of measures that can be implemented in Phase III.

In a first step, a decision needs to be made by the Municipality regarding the district that will be selected for the transformation. This selection will be made taking into account the main characteristics of the district evaluated in terms of the potential to contribute to the transformation of the city, as well as the urgency of the specific needs of each district. The second step on the other hand, corresponds to the diagnosis of the district that will be evaluated for the city. The diagnosis will cover the following aspects; socio-economic analysis (age distribution of the inhabitants, the average household disposable income, the unemployment rate, etc.), morphological aspects (spatial distribution of the buildings, age and use of buildings, etc.), social analysis including aspects such as building owners and public participation, or environmental factors such as the potential for integrating renewable energy sources. Special attention should be paid in the analysis to aspects such as public participation activities developed by the Municipality with regard to the measures that are susceptible to be implemented, since these aspects are critical to identify non-technological barriers and to avoid difficulties during their implementation.

Level 1 indicators are also calculated at district level, when appropriate. This will contribute to the definition of the baseline of the district and will establish a direct link between the changes in the district and the impacts in the entire city, both will be evaluated in Phase VII. The values of these indexes will allow understanding better the context information at district scale with the quantitative values of indicators that are in line with the analysis carried out at city scale in the Phase I of the methodology. Besides, having the specific values of these indicators at district scale will aid to define specific objectives and measures and to stablish the prioritization criteria.

Taking into account all the information gathered at district scale, the energy demand and consumption of the base case scenario needs to be modelled for the district to allow the comparison with the forecast scenarios in the next phases. In this regard, energy planners usually need to combine various tools with different scales, and approaches, which makes difficult to develop a baseline analysis. Over the last 20 to 30 years, many different tools for building energy modelling have been developed. Several reviews, such as (Swan \& Ugursal, 2009) and (Bourdic \& Salat, 2012), describe different modelling approaches that can be used for evaluating the energy consumption of buildings at the district level. In the case of the tools that are designed for building energy modelling, the availability varies depending on the scale of the project. As mentioned by (Martos, Pacheco-Torres, Ordóñez, \& Jadraque-Gago, 2016), the development of tools for evaluating and predicting the future energy consumption of cities and districts will be one of the biggest challenges in the field. However, the situation is much better in terms of the availability and reliability of tools for analysis of single buildings or a reduced building group. There are several tools, such as EnergyPlus and TRNSYS, that are broadly accepted and that allow for a very detailed and dynamic building energy simulation. Other modelling approaches such as BIM models, as well as the life cycle approach, are also identified as useful for this baseline definition stage. All these type of models and approaches need to be combined at this phase to achieve an appropriate analysis of the district.

This baseline analysis combined with the context information and the district scale Level 1 indicators will be used for the definition of the specific objectives of the district. These objectives are the conclusion from Phases I and II. They consist on targets related to improve the environmental, economic and social profile of the district and the city, and can be related to specific measures that are susceptible to be implemented in the district in order to contribute to the compliance of the targets of the city.

\section{Phase III: Definition and analysis of the intervention and scenarios}

The main target of this phase is to define the possible retrofitting scenarios to be considered for being implemented in the selected district according to the city (general objectives) and district needs (specific objectives). These scenarios consist of groups of measures that aid to forecast the impact on the sustainability performance. Before defining the scenarios, it is necessary to decide which measures should be discarded and for that purpose, the potential of each measure with respect to the objectives achievement is analyzed individually.

The ECG is the responsible of these activities. As a multidisciplinary group, it assists the Municipality to facilitate the analysis and the decision-making process. The ECG performs this supported on different recommendations, simulation tools and Indicators. In addition, the Technical and Steering committees from the Municipality can follow and control the process with them.

The measures to be considered are named as Energy Conservation Measures (ECMs). They are defined as "measures that are applied to a building or group of buildings to improve energy efficiency and are life cycle cost 
effective and also they involve energy conservation, cogeneration facilities, renewable energy sources, improvements in operations and maintenance, or retrofit activities" (CITyFiED, D4.18). The catalogue of ECMs proposed in the methodology collects the CITyFiED "Basket of technologies" (CITyFiED, D1.7); a reduced list with the most efficient measures identified within CITyFiED Project approach. They are grouped into different categories: the first two focus on decreasing the energy consumption and the third one promotes the use of renewable energy sources, saving $\mathrm{CO}_{2}$ and Primary Energy.

The steps to achieve by the ECG during Phase III are mainly two. The first one is the measures preselection, which consists of a preliminary choice of measures applying a filter, the specific objectives set in Phases I and II. The methodology establishes the relationships between ECMs and objectives, so it is possible to discard some of them at this early stage. In addition, the results of the Level 1 indicators, the feedback from the citizens (public participation activities) and Municipality, and other aspects identified during the analysis at both scales i.e., nontechnological barriers, financial opportunities, etc., should be considered for this preliminary step.

The second step consist of a feasibility analysis of the preselected measures and the final selection of those that will be part of the retrofitting scenarios. For this analysis, it is necessary to evaluate the environmental, economic, technical and social aspects. In order to guide this study qualitatively, estimations from research on energy and $\mathrm{CO}_{2}$ emissions levels of savings, payback periods, social issues, etc. are included in the catalogue of measures, as well as general recommendations for district retrofitting that affect the measures selection based on CITyFiED experiences (CITyFiED, D2.1 and D6.4) or other sources (STBA).

On the other hand, "Level 2 indicators" defined in the CITyFiED project could be calculated to assess Environmental, Technical, Economic and Social aspects along the application areas, and compare the results obtained for each ECM. The ECG could consider calculating at this stage only the most relevant indicators with respect to the objectives. Finally, previous recommendations and L2 indicators make possible to select a list of the most cost-effective measures ECMs to reach the sustainable objectives that the city desires.

Scenarios are tools to predict the results that could be obtained in case of their implementation. There is a high number of possible ECMs combinations, so it is necessary to generate the scenarios with the information already obtained, considering also experts' intuition and experience. The procedure proposed starts defining those measures considered as indispensable to proceed with the intervention as the starting point. Then, the rest of scenarios are defined increasing the performance from that set point. They are sorted in three groups depending on the $€ / \mathrm{Kg} \mathrm{CO}_{2}$ savings ratio estimation - basic, efficient and advanced.

When each scenario is specified, it is necessary to recapitulate the information to analyze the retrofitting scenarios as units in next Phase IV, due to the result of the combination of some ECMs could be different from the addition of the individual results.

\section{Phase IV: Prioritization and selection of the intervention scenario}

The objective of this phase is to identify the most convenient solution from the potential scenarios. The ECG expertise, Level 2 indicators applied to the alternative scenarios and multi-criteria analysis, are the basis for the decision-making process. Finally, the discussion is expected to be carried out between technicians, citizens and local authorities.

The first step is focused on the evaluation of alternative scenarios. Here, the results of the different scenarios defined in the Phase III are evaluated considering the economic, social, technical and environmental dimensions. With this aim the defined Level 2 indicators at project level are evaluated for each scenario following different approaches, such as energy simulation tools for district renovation, building information modelling (BIM), geographic information systems (GIS) tools and the Life Cycle Analysis (LCA).

The results obtained from this analysis are used in the scenario prioritization step. It is necessary to take into account that the prioritization of strategies and scenarios needs to be based on the evaluation of a number of criteria that cannot be optimized simultaneously. Therefore, in the context of urban energy planning it can be said that there is no a unique optimum scenario within the set of alternative options for achieving a specific goal for the city.

Different methodologies are needed to support the decision-making once the evaluation studies have been carried out. With this aim, Multi-criteria Decision Analysis (MCDA) offers a wide range of methodologies and tools to 
support decision-makers, allowing the combination of their own preferences with the data available (from modelling or not) to reach their own conclusions in a structured and consistent way. In the last few decades, the number of MCDA methods has substantially increased and nowadays, there are hundreds of methods available (Hobbs \& Horn, 1997). Many studies and different classifications of these methods can be found in the literature. In the review carried out by (Pohekar \& Ramachandran, 2004), an assessment of more than 60 studies regarding the application of multi-criteria decision making to sustainable energy planning is presented. In the same way, the study by Diaz-Balteiro et al. (Diaz-Balteiro, González-Pachón, \& Romero, 2016) offers an extensive review of 271 papers using MCDA methods for measuring systems sustainability. The study shows that the use of methodologies such as the AHP developed by Saaty (Saaty, 1980) is in general, increasing in recent years. From the literature review, it can be concluded that using multi-criteria analysis in the context of urban renovation has attracted the attention of decision makers for a long time and that although there is not a specific method that can be prioritized, some of them seem to be more appropriate if we consider the development of the last few years. For CITyFiED methodology, the method proposed for the prioritization, in order to combine both the quantitative results of the scenarios and the qualitative relevance of each criterion, is the AHP.

In the first step of the application of the AHP method, a disaggregation of the problem has to be done defining a hierarchy of the interrelated elements, identifying the general objective, the specific objectives or criteria and the potential alternatives or scenarios.

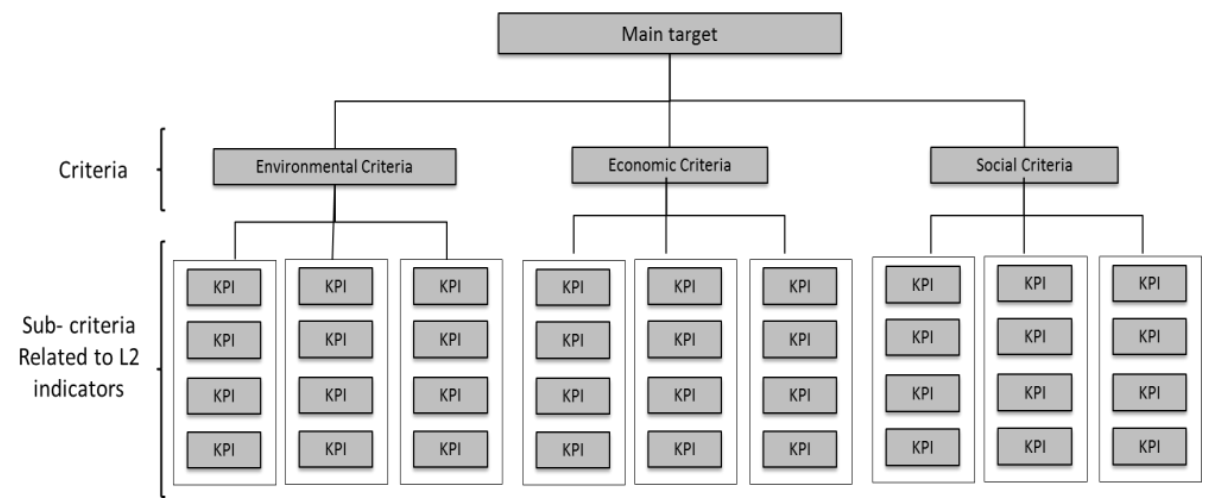

Fig. 5: Hierarchical problem structuring example

The specific objectives defined at district scale in the Phase II can be used as the basis to define the main criteria for the prioritization. In the same way, the sub-criteria that contribute to the consecution of the specific criteria can be related to the Level 2 indicators evaluated for each scenario. Therefore, for the identification and selection of the prioritization criteria, a clear connection can be made at this stage with the Level 2 indicators. This decision will be taken by an open dialog and consensus between technicians and local authorities. Including public participation at this stage of the process and taking it into account in the decision making is really relevant for engaging citizens in climate challenge.

In a second step, the weighting of each criterion needs to be also defined following the same procedure. Finally, through the complete application of the AHP method, the evaluation of the performance of each scenario for each prioritization criteria is carried out. The scenario with the highest result is identified as the most suitable one.

\section{Phase V: Strategies for Sustainable Urban Renovation (SSUR)}

The main target of this phase is to define the set of Strategies for Sustainable Urban Renovation (SSUR) where the ECMs of the selected scenario are further developed. In addition, these strategies should be integrated within the Strategic Urban Plan of the city, as a long-term vision plan for the city.

Local authorities, especially the Technical committee - and the ECG if it is desired by the Municipality - are in charge on the development of the SSUR, which is understood as a highly participatory management tool, that is to say this document is a process, not a project. It contents all the development of the previous phases.

First of all, the documents presents the causes for developing a Strategy for Sustainable Urban Renovation, the past of the city is explained as well as current situation. This means to give the answers to the questions where do we come from? And where are we? The vision of the city from Phase I is defined, by explaining the local authorities' motivations for implement sustainable strategies; Where do we want to go? 
Secondly, the document exposures the work done by the ECG together with the Technical committee regarding the city analysis and diagnosis, the scope definition, to be specific, the district selected. Moreover, it is also presented the scenarios evaluation and the final selection.

Finally, it presents the Strategy which compromises "a co-ordinated set of participatory and continuously improving processes of analysis, debate, capacity-strengthening, planning and investment, which integrates the economic, social and environmental objectives of society, seeking trade-offs where this is not possible" (OECD 2001).

The Strategy does not end with the exposure of the selected scenario in Phase IV, but also it will guide and coordinates the process during its implementation through a Strategy Implementation Plan (SIP), which will detail the intervention actions to be applied in the district, the schedule which includes a temporary planning for each actuation line during the period of SIP's life, and finally the budget of the implementation plan.

On the other hand, one of the most important aspects during the definition of the strategies is the need to guarantee its success through not only ensuring an effective participation of the citizens (CoM, 2010), but also the identification of the non-technological barriers and the potential business models that will enable the realization of specific strategies.

Non-technological aspects, such as financial, organizational, legal, cultural and social, have be explored during the process (from phases I to IV). For that, a participatory methodology was designed in CITyFiED project to involve key stakeholders, this procedure is based on interviews that should be adapted according to the specific of the cities to identify the barriers and potential for energy efficient retrofitting.

CITyFiED Methodology will proposed also a mechanism to analyse possible business models and investment schemes for the cities to identify the most convenient, in order to minimize gaps and risks of the suggested strategies.

Last but not least, the document Strategies for Sustainable Urban City Renovation should detail recommendations to ensure that the skip from Planning to Implementation phases will be done properly. In that sense, the Strategy should define the Method of Procurement (MOP), detailing the factors that influence it, such us project characteristics, cost issues, timing, external factors, client resources, etc.

In addition, recommendations for the Monitoring and Impact Assessment Evaluation Phase (Phase VII) are also given, regarding the need to develop a Sustainable Evaluation Plan (SEP), which coordinates the activities and guarantee the city goals achievement. The SEP, understood as a protocol to evaluate the sustainability and quality of the interventions at district level after their implementation (Phase VI). It should be based on the evaluation of the energy performance and energy savings through well-known Measurement \& Verification (M\&V) protocols (e.g. IPMVP, FEMP or ASHRAE), the economic issues, such as return of investment (ROI) and cost effectiveness of the solutions, the Quality control of the interventions, the Social acceptance and a Life Cycle Analysis (LCA), to evaluate the $\mathrm{CO}_{2}$ emission reduction.

\section{Phase VI: Execution plan}

The strategies from the SSUR document are implemented in this phase, according to the recommendations from planning to implementation and the SEP guidelines pre-defined in Phase V. The detail definition of these activities depend in other aspects, i.e., technical definition, that could not be defined before the SSUR. Therefore, these issues and specific guidelines to put into practice the strategies - delivery method, procurement process, risk allocation, etc. - are further developed in Phase VI, acting as an overall Execution Plan.

The Municipality, the ECG and financial institutions are involved in these activities, as well as building owners and citizens. Contracting parties group are the principal figure for the execution of works, including designers, ESCOs, energy suppliers, technology providers, etc. Their participation starts at this stage because in public procurements they usually have to be selected through a tender/bidding process.

Part of the execution procedure is to clarify the financial resources to put into practice the SSUR according to the Business model identified and the ownership of the properties to be renovated. In the case of public ownership of the buildings the Municipality usually acts as financier; while in private ownership the ESCO business model is highly recommended. The most relevant public financing mechanisms are grants, interest subsidies, revolving funds and financial instruments (Núñez et al., 2013). 
A Project Delivery Method has to be identified by the Municipality to define the contracting formats between the stakeholders to achieve the delivery (CMAA, 2012). There are different methods but the most recommended is the Integrated Project Delivery (IPD), defined as "a collaborative alliance (...) that harnesses the talents and insights of all participants to optimize project results, increase value to the owner, reduce waste, and maximize efficiency (...)" (AIA, 2007). Its principles focus on the collaboration since the beginning of the project to avoid future problems and the share of risks and responsibilities between the stakeholders.

The Procurement starts after, to define the process of services or works acquisition for the implementation of the strategies. In public procurement is usually mandatory to select the entities through a tender/bidding process. Attending to the scope the Municipality could contract just the implementation (Build), if it wills to develop the technical definition of the strategies. By contrary, it could offer the design and the execution to different entities (Design / Build) or the same one (Design + Build). The winners of the tender will be part of the Contracting parties group. The tender documents can be prescriptive if the requirements are technically specified or performance based, and in the second case also related to reduce the environmental impact what is known as sustainable procurement (Pless et al., 2012).

In parallel to the two previous activities, the district and strategies need to be further developed into detail from the technical and economical perspectives. It is recommended to support the technical definition on BIM approach that allows to address responsibilities, layouts, schedule, risks, etc. in a collaborative framework, and it is identified as the most adequate to maximize the benefits of IPD principles (AIA, 2007). Depending on the procurement selected the technical definition of the strategies is performed before or after tender/bidding processes.

Another relevant aspect is to include a Risk Management Plan in the context of IPD, to eliminate or reduce the risk that could be avoided, establish mitigation actions to reduce the probability of occurrence, or develop contingency strategies for those risks that could be predicted or avoided (Association of Project Managers, 2000).

The follow-up strategy during the execution of works (Phase VI) and evaluation phase (Phase VII) is the SEP already pre-defined in SSUR document and that is completed by the Monitoring and Technical Committees during this phase once the technical definition of the strategies is achieved. The follow-up of the intervention should be reported monthly or bimonthly, and gathered in annual reports in the SSUR. In parallel, regular meetings should be established between the actors involved to control and make decisions and changes approval.

\section{Phase VII: Monitoring and impact assessment evaluation}

The main objective of this last phase is to evaluate and monitor the strategies implemented during the previous phase, because at this stage, actions and construction works are completed, so it is necessary to assess the impact in the sustainability at district and city level, and finally to deploy corrective actions if the impact obtained it is not the expected. So that, the objective of the monitoring plan is twofold, evaluate the performance and impact of the strategies at district level (Level 2 indicators) and city level (Level 3 indicators).

Within CITyFiED project a Sustainable Evaluation Plan (SEP) has been defined (CITyFiED, D4.10) in order to assess the impact in the sustainability at district/project level. The objective of the plan developed was to reduce the complexity of some existing standards such as BREEAM and/or LEED and integrate new pillars in the evaluation according to the new trends, i.e. ICTs and LCA. Thus, Fig. 6 illustrates the protocol proposed, including its pillars: Energy, ICTs, Quality control of interventions, Economic evaluation, Social acceptance and LCA.

As it can be seen in Fig. 6, the left hand side represents the analysis before the implementation (Baseline calculated during Phase II), meanwhile the right hand side denotes the assessment after intervention; at the top, Level 2 indicators (KPIs) give an objective the framework for calculating the evidences of assessment, and at the bottom, LCA covers the project from the beginning to the end of the works. In the middle, the ECMs appear, which refer to the interventions themselves.

Starting from the left hand side, it is shown that the energy concerns begin with social awareness, both at citizens' level and at organizational level (public authorities, sustainability plans, etc.). Collecting all these aspects through metering determines the retrofitting strategy as well as the best ECM for the specific building or district.

On the right hand side, the status after the execution phase is evaluated. The main aspect is the energy performance in terms of energy savings, which is partially achieved by the investment in new ICT technologies (digital homes), 
and the ECMs. To ensure the expected energy savings, the ECM has to be implemented in the correct way following Quality Control of interventions rules during Phase VI. These energy savings drive to cost savings at consumption. And thanks to the energy efficiency, less invoice costs and new technologies, together with the involvement of citizens, the social acceptance of these solutions is increased.

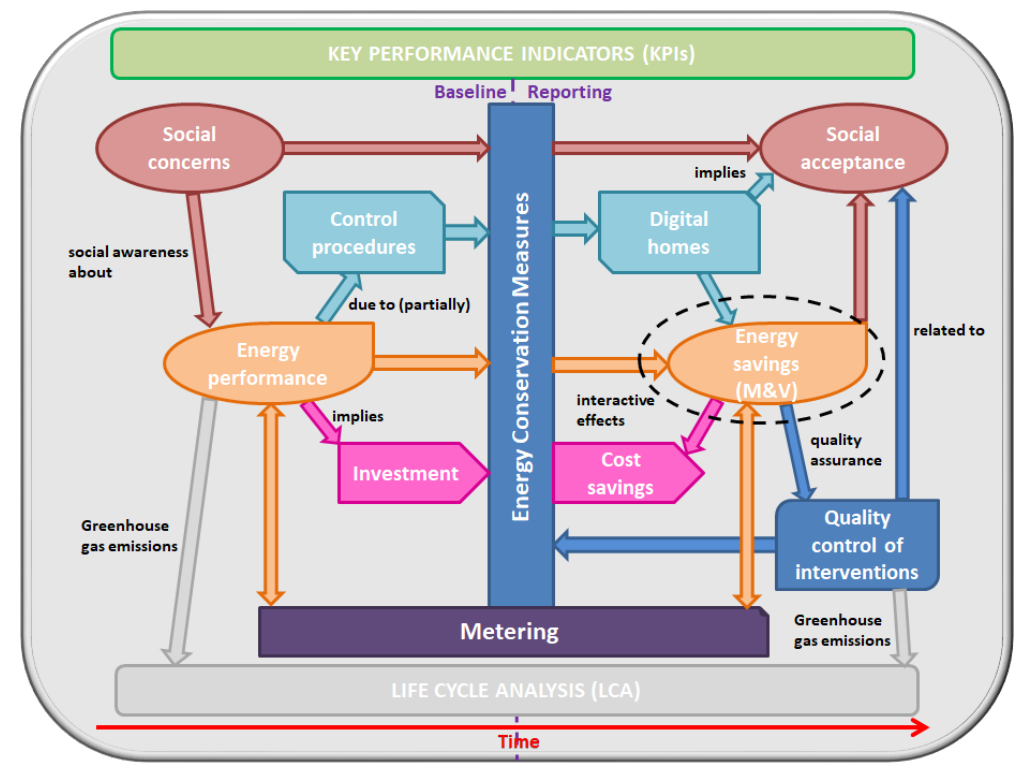

Fig. 6: CITyFiED Sustainability Evaluation Plan (SEP) at district level

The CITyFiED Methodology, as a systematic process, considers that if the results obtained after the deployment of the SEP not achieve the expected results at district level, corrective actions should be applied, starting from the analysis of SSUR if necessary. The deviation between the expected results and the obtained ones, is part of the evaluation and means that the General and Specific objectives defined during Phase I and II are only partially achieved. This experience is part of the correction actions for future city renovations that all the stakeholders from the Municipality should consider, too.

In the same way, the impact of the interventions at city level will be evaluated. For that purpose, there was defined a set of indicators (Level 3: Impact Assessment Indicators at city level) in order to evaluate the following categories of impacts after the strategy implementation: Energy impact: Energy savings obtained; Environment impact: $\mathrm{CO}_{2}$ emissions avoided with the intervention; Economic impact: investment mobilized and its return to the users, companies and municipalities as well as benefits of retrofitting to the users associated to the cost savings; and Benefits for SMEs.

\section{Conclusions}

This Methodology is a holistic procedure for the city renovation at district level that considers a multi-criteria perspective. The integration of the three levels of indicators supports the diagnosis, selection as well as final evaluation of measures and retrofitting scenarios during the project, serving as a control and decision-making tool. The CITyFiED project aims to enable the replicability and mass-market deployment of energy-efficient retrofit of districts. The approach and the indicators were conceived and are being refined considering as a reference the large CITyFiED demonstration cases in the cities of Lund (Sweden), Laguna de Duero (Spain) and Soma (Turkey), including decision-making processes and business models. The approach is also being validated through the active participation of the CITyFiED network of cities, assuring its flexibility and adaptability to different European cities. Then, the future work is to complete its development, continue its external validation activities with the CITyFiED network of cities and extract conclusions.

Acknowledgments: This project has received funding from the European Union's Seventh Programme for research, technological development and demonstration under grant agreement $\mathrm{N}^{\circ} 609129$. The authors would like to thank the rest of the partners of the CITyFiED project for their help and support. 


\section{References}

American Institute of Architects California Council, 2007. Integrated Project Delivery - A Working Definition.

ASHRAE Guideline 14-2002, June 2002. Measurement of Energy and Demand Savings, ASHRAE Standards Committee,

Bourdic, L., \& Salat, S., 2012. Building energy models and assessment systems at the district and city scales: a review. Building Research \& Information, 40(4), 518-526.

Broere, W., 2015. Urban underground space: Solving the problems of today's cities. Tunnelling and Underground Space Technology.

CITyFiED Consortium, 2017. CITyFiED Project, Grant Agreement no. 609129.

Covenant of Mayors, 2010. How to develop a Sustainable Energy Action Plan (SEAP) - Guidebook. Belgium: Publication Office of the European Union.

Diaz-Balteiro, L., González-Pachón, J., \& Romero, C. (2016). Measuring systems sustainability with multi-criteria methods: A critical review. European Journal of Operational Research, 258(2), 607-616.

Dijkstra, L., Poelman, H., 2012. Cities in Europe. The new OECD-EC definition.

Efficiency Valuation Organization. (September 2010). International Performance Measurement and Verification Protocol, Concepts and Options for Determining Energy and Water Savings, Volume 1, EVO 10000 - 1:2010.

Dodd, N., Garbarino, E., Gama, M., 2016. Green Public procurement Criteria for Office Building Design, Construction and Management. Joint Research Centre. EC.

Eurostat. (2016). Urban Europe. Statistics on cities, towns and suburbs.

FEMP M\&V Guidelines, The M\&V Guidelines: Measurement and Verification for Federal Energy Management Projects, U.S. Department of Energy, Version 3.0.

Hernández, J.L., García, J., Zubia, C., Pablos, L., Cueva, F., Vasallo, A. Novel sustainability assessment procedure proposal for Smart Cities, Central Europe towards Sustainable Building 2016 (CESB 2016), Prague, Czech Republic, 22-24 June 2016.

Hobbs, B. F., \& Horn, G. T. F. (1997). Building public confidence in energy planning: a multimethod MCDM approach to demand-side planning at BC gas. Energ. Pol., 25(3), 357-375.

Martos, a., Pacheco-Torres, R., Ordóñez, J., \& Jadraque-Gago, E. (2016). Towards successful environmental performance of sustainable cities: Intervening sectors. A review. Renewable and Sustainable Energy Reviews, 57, 479-495.

Núñex, F., Olivero, S., Medarova-Bergstorm, K., Rizos, V., 2013. Financing models for smart cities. Guidance document. Financing Working Group. Smart Cities Stakeholder Platform.

OECD. (2001). Strategies for Sustainable Development. The DAC guidelines. France. pp. 16.

Petts, J., Leach, B.. 2000. Evaluating Methods for Public participation: Literature Review. R\&D Technical Report E135. Bristol: Environment Agency. pp. 26-33.

Pohekar, S. D., \& Ramachandran, M. (2004). Application of multi-criteria decision making to sustainable energy planning A review. Renewable and Sustainable Energy Reviews, 8(4), 365-381.

Saaty, T. (1980). The analytic hierarchy process. New York: McGraw-Hill.

Smart Cities Council, 2013. Smart Cities' readiness guide. The planning manual for building tomorrow's cities today.

Swan, L. G., \& Ugursal, V. I. (2009). Modeling of end-use energy consumption in the residential sector: A review of modeling techniques. Renewable and Sustainable Energy Reviews, 13(8), 1819-1835.

The Association of Project Managers, 2000. Project risk analysis and management. Great Britain.

The Construction Management Association of America (CMAA), 2012. An owner's guide to project delivery methods.

United Nations. (2014). World Urbanisation Prospects.

URBACT II. (2015). Sustainable regeneration in urban areas

World Bank, 1999. World Development Report 1999/2000: Entering the 21st Century. Ch. 6.

Web References:

BPIE EU Buildings database <http://ec.europa.eu/energy/en/eu-buildings-database> September 2017

Energy eficiency directive <https://ec.europa.eu/energy/en/topics/energy-efficiency/energy-efficiency-directive> March 2017

EnergyPlus. Building Energy Simulation Software. Retrieved from 〈http://apps1.eere.energy.gov/buildings/energyplus/> September 2017

Technical Committee ISO TC/268

<http://www.iso.org/iso/home/standards_development/list_of_iso_technical_committees/iso_technical_committee.htm ?commid=656906>, March 2017

PLEEC Project <http://model.pleecproject.eu $>$ March 2017

Urban Regeneration Decision Tool by World Bank <http://urban-regeneration.worldbank.org/about>, March 2017

Responsible Retrofit Guidance Wheel of the Sustainable Traditional Buildings Alliance (STBA) <http://responsibleretrofit.org/wheel/> September 2017 